\title{
Fuzzy Automating Strategies of Emergency Operation for SGTR Accident in VVER 1000 Nuclear Reactor
}

\author{
Ebrahim Modarres*, Faridoon Shabaninia
}

Shiraz University, Iran

Copyright (C) 2015 by authors, all rights reserved. Authors agree that this article remain permanently open access under the terms of the Creative Commons Attribution License 4.0 International License.

\begin{abstract}
Steam generator tube rupture is one of the most important accidents that is focused nowadays. In this work, the automating strategies of emergency operation are developed for SGTR accident in VVER 1000 nuclear reactor. After recognizing accident, reactor must be shut down and controlled properly in order to reach safe state of that. In control, cooldown is planned by establishing operational goals, forming strategies, and specifying operational means and constraints . According to operation manual reactor, cooling is done by means of releasing steam from steam valve BRU-A on steam generator. The reference set points of the controlled parameters are adaptively adjusted through fuzzy reasoning for optimal cooldown and depressurization. These strategies can make emergency operation optimal, and as well they considerably lengthen the operator response time. The validation of the proposed strategies was carried out using the micro-simulator with steam generator tube rupture(SGTR) events. The results indicate that the automation emergency operation successfully drives the plant at full power to a cold shutdown state with all the operational constraints satisfied. Analysis of result shows us:A. pressure of first loop wouldn't exceed primary value during accident. Maximum of pressure of reactor in second loop on steam generator " $\mathrm{B}$ " is 71.91 barg. B. maximum temperature of fuel rods and fuels don't exceed primary values. Maximum and minimum temperature of fuel rods are $788^{\circ} \mathrm{C}$ and $546.95^{\circ} \mathrm{C}$ respectively.
\end{abstract}

Keywords VVER 1000, SGTR, Automation, Simulator, Fuzzy

\section{Introduction}

In large and complex machine systems, many operations have become more difficult for users, resulting in a greater potential for operation errors. Such mistakes may cause serious accidents, especially in aircraft industries and power plants. Hence, many machine systems have been automated through the introduction of modern computer and information technologies [1].When a nuclear power plant (NPP) is in a normal state, In order to maintain plant, routine monitoring and control status are performed by the operator. These tasks are not too complex that they can cause much cognitive burden or human error on one or more operators. When plant is in an abnormal state, most operators would feel a physical/cognitive overburden [2]. Operating procedures exist for virtually all aspects of reactor operation including emergency response. However, these procedures, which are written sequentially, place severe burden on operators as they have to identify and evaluate the symptoms to be applied and they have to interpret the procedures to carry out the recommended actions. Thus, the operators that are trying to apply current procedures to difficult situations may still burden significant strain. The result is a corresponding potentially negative impact on plant safety. By analysing of event reports it is shown that the most commonly cited cause of the events in the United States is plant operating procedures. In fact, procedural problems as causal factors in $69 \%$ of the reported events have cited by operators [3].The expert systems for procedure tracking are being developed or were developed in the nuclear field in order to overcome these procedural problems [4]. In addition, the feasibility of the operating procedure automation was investigated with its costs and benefits [5], and some researches has been done on operation automation [6]. An especially high level of plant automation has been achieved in Japanese NPP's where the range of automation has been extended in normal operation, plant startup and shutdown, abnormal conditions, and accidents [7]-[9]. Decision-making and control are proposed in order to develop the automating strategies by restructuring the existing emergency operating procedures (EOP's). In this study, there are two valuable points. First one is that it can make emergency operation optimal in view of the material aspect of the reactor vessel. For this purpose, fuzzy inference helps us to cooldown and depressurization in emergency operation. Second, safety and operational margin during post-accident operations would be greater by the automation of emergency operations.

This paper is organized as follows: in Section II, 
Description of VVER 1000 plant. In Section III, we outline an analysis of the current EOP's of a PWR(VVER 1000). In Section IV, the detailed automating strategies of emergency operation are presented. The validation of the proposed strategies during a steam generator tube rupture (SGTR) accident is demonstrated using the micro-simulator in Section V. Finally, in Section VI, conclusions are presented.

\section{Description of VVER Plant Model}

\section{BNPP Plant}

The reference power plant for this analysis is Unit 1 for BNPP. The VVER 1000/V446 is a pressurized water reactor (PWR) that produces $3012 \mathrm{MW}$ thermal power and generates $1000 \mathrm{MW}$ electrical power. It has four coolant loops, four reactor coolant Pumps, horizontal SG on each loop. The behaviour of horizontal steam generators is very different in comparison to western types which are vertical SGs.The unit 1 has the standard emergency core cooling system (ECCS) including high\&low-pressure injection system and four hydroaccumulators. Primary pressure maintenance system consists of pressurizer, surge line, spray line and pulse safety facility. Reactor pressure vessel has four inlet and four outlet nozzles with a higher elevation than the inlet nozzles. Reactor coolant system (RCS) transports heat from the reactor core to the SGs that provide steam to the TG through the main steam lines. Four primary coolant loops have a common flow path through the reactor vessel. Each RCS loop includes a horizontal SG and a MCP. The primary coolant flows from the reactor outlet nozzles to the SGs and then is pumped by MCP to the reactor inlet nozzles. Steam produced in the secondary side of the SG passes through the MSIVs and flows into the common steam header (CSH) of the turbine. The steam generators have a very important role for safety and reliability of VVER nuclear power plants. SGs safety devices are:

a. Pilot-operated SG pulse safety device (PSD) that opens at 8.24 MPa and closes at $6.87 \mathrm{MPa}$.

b. Working SG PSD opening and closing pressure is 8.44 and $6.87 \mathrm{MPa}$, respectively.

c. Steam dump valve to the atmosphere (BRU-A) with opening and closing pressure at 7.15 and $6.27 \mathrm{MPa}$, respectively.

d. Steam dump valve to the turbine condenser (BRU-K) with opening and closing pressure of 6.67 and $6.27 \mathrm{MPa}$ respectively.

\section{e. Fast acting steam isolating valve (BZOK).}

The steam generators are fed by two different feedwater systems. Auxiliary feed water (AFW) and emergency feed water (EFW). AFW and EFW provide feed water to SGs during transient. Four diesel generators provide electricity for AFW independently if the loss of onsite power occurs in plant. Otherwise, EFW is activated and injects water to SGs. The turbine is designed to operate at saturated steam in a single unit configuration. TG produces 1000MW of electric power when the reactor nominal thermal power is $3012 \mathrm{MW}$. Turbine consists of one high pressure and three low pressure turbine. At first, steam enters to high pressure. After it passes through moister separator reheated, and then enters the three low pressure turbines.[19]

\section{Simulator}

Micro-Simulation Technology has recently completed PCTRAN/VVER for the Russian-designed VVER1000 and newer G3 models.

Since the early V320 models built and operating in Russia and a number of east European countries, there are improved AES90 and 91 models built in Iran, India and China. The latest AES92 meets European Design Criteria for the so-called "Generation III" criteria with improved passive cooling systems and debris collating devise. It will be the main model for future construction. For educational purpose, the PCTRAN VVER1000 is not modeled after any specific one of the above. Instead a "generic" to accommodate all by making greatest effort in adjusting the plant input data so that the same software could simulate any one of them.

In the NSSS mimic below (fig.1), a single loop with the pressurizer is modeled separately from the other three loops lumped together. Plant data parameters controlled by the users would define the model to represent a specific plant.

There are full control systems for the reactor, pressurizer and steam generators. The plant can be controlled either by manual or automatic. In automatic it can be either turbine-driven or reactor driven. For emergency core cooling, Model V320 is provided only with high and low pressure nitrogen-filled tanks. Later AES series have added high-pressure injection pumps. Here in PCTRAN we provide both in the left side panels in the mimic. So the same software can simulate either plant simply activate or disable certain systems. The plant size and power between each model are also different. That is accommodated by various input data sets. 


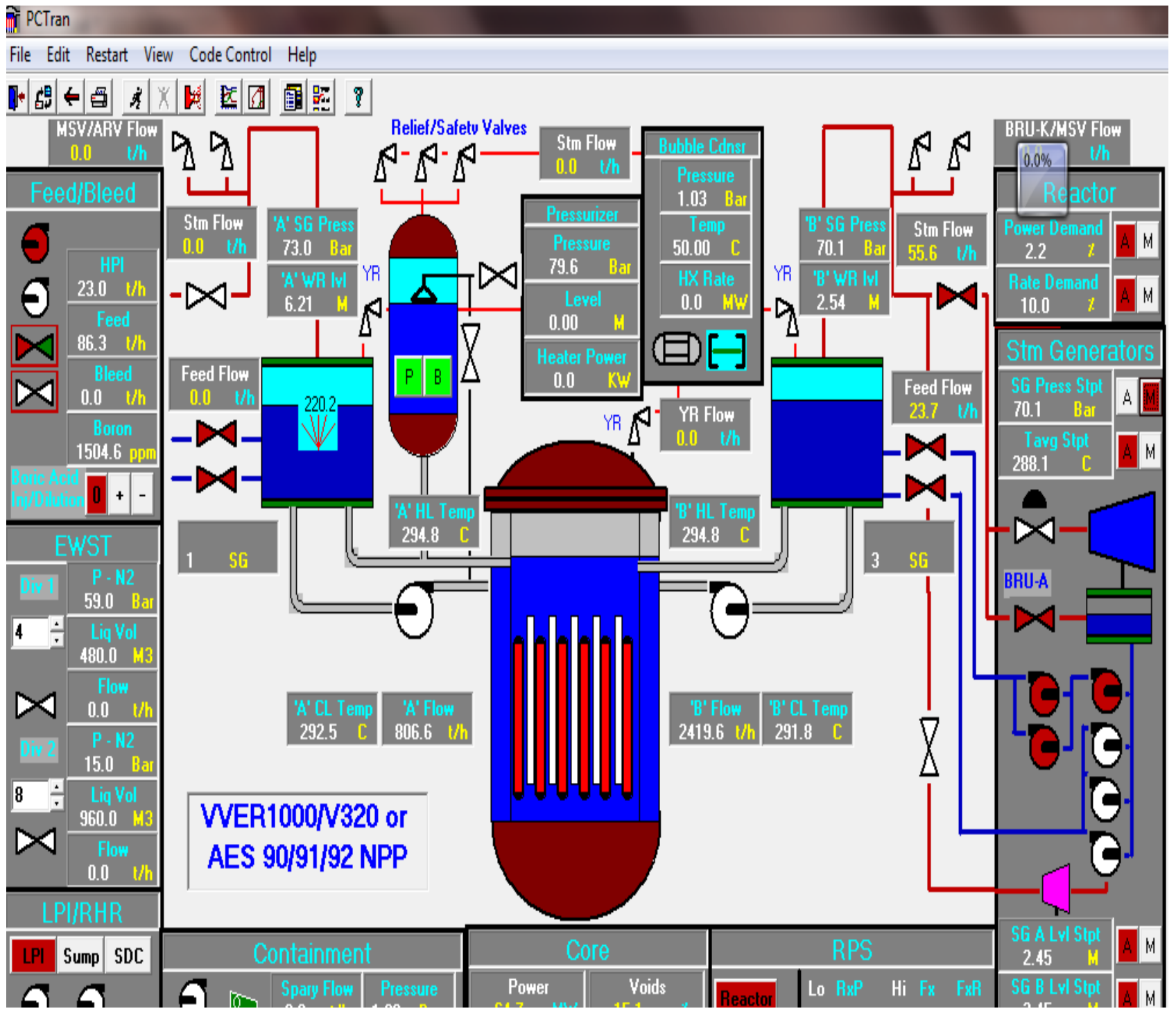

Figure 1. PCTRAN VVER1000 Micro-Simulation

\section{Emergency Operation in a VVER 1000 Nuclear Reactor}

The operating procedures for NPPs consist of five components, as shown in Fig. 2. Operators in a NPP operate the plant according to the system operating procedures( SOP) and the general operating procedures (GOP) in normal state. When an alarm occurs, the operators monitor necessary signals and manipulate appropriate devices through the Alarm Recovery procedures(ARP). When multiple alarms occur, the operators act through the Abnormal Operating procedures(AOP). If the reactor is tripped and safety injection (SI) is operated in an abnormal state, the operators monitor necessary signals and manipulate necessary devices to put the NPP in a hot standby (HSB) state through the Emergency Operating Procedures( EOP).

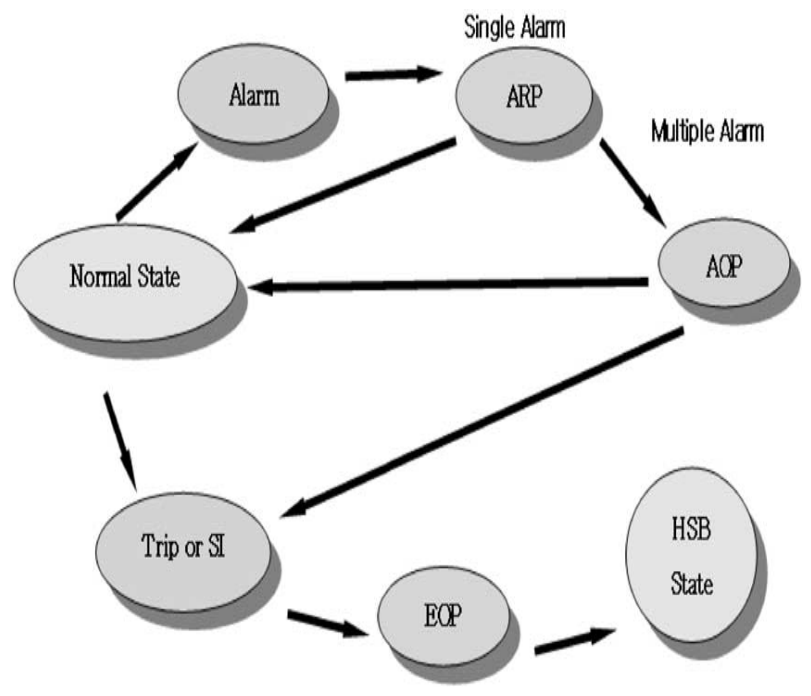

Figure 2. Procedure analysis 
In this work, the target operating procedures of the automating strategies are the EOP. The EOP consists of four large categories, as shown in Table 1.

Table 1. Emergency operating procedure analysis

\begin{tabular}{|c|l|}
\hline E-0: Reactor trip or SI & $\begin{array}{l}\text { ES-0.0: Accident diagnosis } \\
\text { ES-0.1: Action for reactor trip } \\
\text { ES-0.2: Cooling by natural cycle }\end{array}$ \\
\hline & $\begin{array}{c}\text { ES-1.1: SI termination } \\
\text { ES-1.2: Cooling and depressurizing } \\
\text { after LOCA } \\
\text { E-1: LOCA of primary or } \\
\text { secondary loop }\end{array}$ \\
$\begin{array}{c}\text { ES-1.3: Transition to cold leg } \\
\text { recirculation } \\
\text { ES-1.4: Transition to hot leg } \\
\text { recirculation }\end{array}$ \\
\hline E-2: Failed S/G isolation & $\begin{array}{c}\text { ES-3.1: Cooling after S/G tube rupture } \\
\text { using } \\
\text { E-3: S/G tube rupture } \\
\text { reverse supplement method } \\
\text { ES-3.2: Cooling after S/G tube rupture } \\
\text { using } \\
\text { B/D method }\end{array}$ \\
\hline
\end{tabular}

The EOP outlines the procedures for emergency situations such as LOCA or SGTR. If the reactor is tripped or SI begins, operators should operate the plant according to the E-0 procedure. The E-1 procedure is for LOCA, and the E-2, E-3 procedures are for SGTR.

In the context of this work, we have interest in the SGTR which is called the E3 procedure, described below.

Although the main purpose of E3 is to stop the radioactive release from the RCS to the secondary circuit and eventually to the outside-of the containment building (phase I), it is also designed to reach a long term safe state (phase II). Phase I is achieved by eliminating the pressure difference between the primary and secondary side. Thus, depressurization of the RCS is necessary and, in order to avoid saturated conditions, a cooldown of the primary circuit is required. Phase II includes actions taken to allow the actuation of the residual heat removal system to go to a cold shutdown safe state. For the methodology demonstration purposes of this work, we have only considered phase I.

When either the reactor protection system limits or the safeguards actuation system limits are exceeded in a VVER 1000, an emergency operation starts as shown in Fig. 3. First of all, the automatic actuation of the protection systems is immediately confirmed. Then it is decided whether the plant trip is a planned one or results from an accident, by checking the safety injection (SI) status. If the trip is a planned one, routine shutdown actions are carried out. Otherwise, an initiating event is identified through a symptom-based diagnosis and, according to the identified event, the response actions are taken on the basis of two concepts [10]. The first is the optimal recovery concept where the plant is operated in the direction of maintaining it in an optimal end state within the range of minimizing radiological releases or equipment degradation. Second is the functional restoration concept where the critical safety functions (CSF's) are always monitored to prevent uncontrolled release of radioactive materials from the NPP's. When there is a challenge to any one of the CSF's, the optimal recovery operation is interrupted to address a more urgent safety concern, and function-related restoration strategies for responding to emergency transients are established. With the response actions taken, the plant is cooled and depressurized in order to make repairs and ensure no further radiological releases.

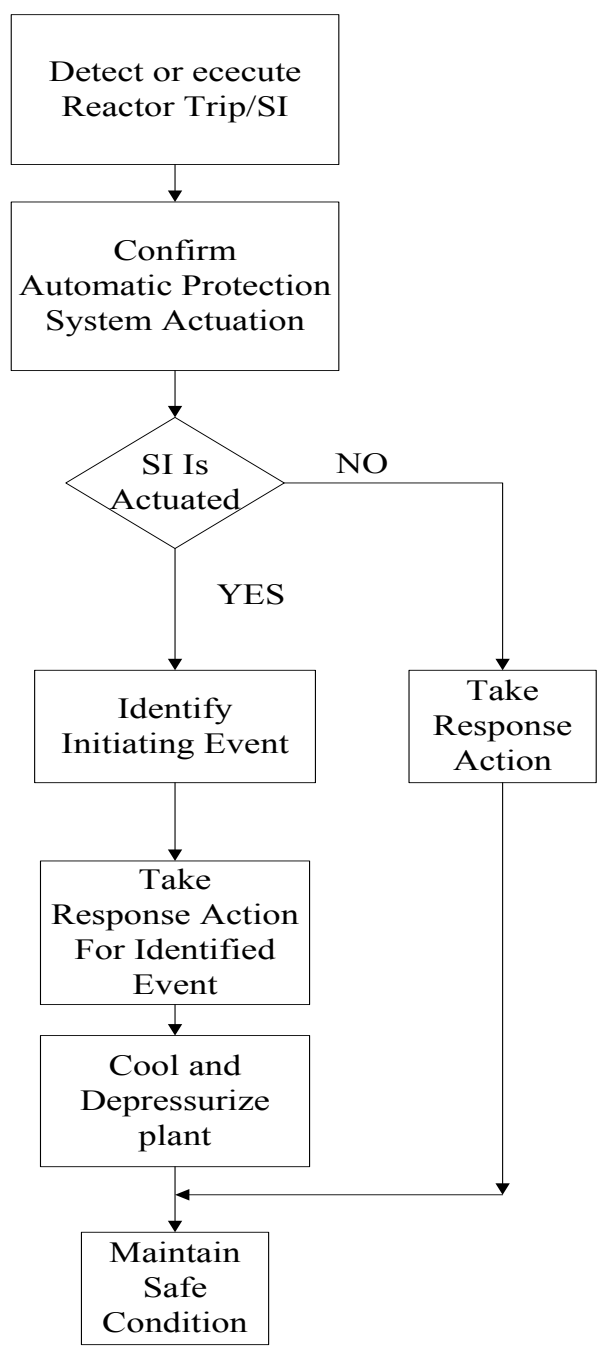

SI:Safety injection

Figure 3. Structure of emergency operation

Emergency operations are composed of monitoring, decision-making, control, and testing. The automation of tests is excluded from the scope of this study because it cannot be achieved without the full automation of experimental test processes in various laboratories. The main activities of monitoring are perception and judgment. This monitoring can be classified into two categories as follows: simple monitoring is a simple observation of parameter values or equipment/component status by the human sensory systems, and cognitive monitoring is an observation that needs complex inference such as the interpretation of the time trends of operating variables or the prediction of their future trends. 
The EOP's require decisions which must be made on a plant- and event-specific basis. These decisions include the diagnostic tasks required to perform appropriate optimal recovery operations and the selection of proper post-SGTR cooldown methods during an SGTR scenario [15].

Control is a direct action that aims at driving the plant to a desired state. Control can be classified into two categories as follows: binary control is a selective action between binary states such as the open/close of valves or the start/stop of pumps; and analog control is a complex action to control process variables such as temperature, flow, pressure, and level. Even if this action seems to be qualitative, the actual tasks for analog control are the proper manipulation of valves/pumps or the adjustment of control valves in most cases.

In the activities described above, there are no problems in the automation of simple monitoring and binary control provided that fault-tolerant software and hardware become available. Therefore, this paper will focus on the automation of cognitive monitoring and analog control as well as the automation of decision-making points for optimal shutdown.

\section{Automating Strategies of Emergency Operation}

Decision-Making

There are some decisions that are required in order to track emergency operation. These decisions consist of the symptom based diagnostic tasks required to proceed to the appropriate optimal recovery operations and the key utility decision points that must be made on a plant- and event-specific basis.

Symptom-Based Diagnosis: After confirming the automatic actuation of the reactor protection system or the safeguards actuation system, symptom-based diagnoses should be made to select the next appropriate operations. In order to automate these diagnostic tasks, diagnostic trees are established through the knowledge elicitation from the senior reactor operators (SRO's) who are familiar with emergency operations. The diagnostic knowledge was elicited on the basis of the three following rules [11]. The first is the hypothesis and confirmation rule. That is, the plant symptoms which enable the operator to hypothesize a certain event has occurred, as well as those which supplement his hypothesis, were identified. The second is the exclusion rule by present symptoms. Some symptoms contradictory to a certain event were identified so as to exclude the hypothesis that the event has occurred if one among those identified symptoms is present. The third is the exclusion rule by absent symptoms. Here, some symptoms essential to a certain event were identified so as to exclude the hypothesis that the event has occurred if one among the identified symptoms is absent.

Fig. 4 shows the diagnostic tree of an SGTR event. Because this tree is composed of logic gates, it is comprehensible and easy to implement using computer programming. However, some qualitative expressions (e.g., uncontrolled decreasing pressure or increasing pressure) are used in the symptoms. These expressions are also related to cognitive monitoring. In the proposed automating strategies, fuzzy membership functions are used to evaluate qualitative symptoms by adopting the algorithm embedded in the Operator Aid SYStem (OASYS) developed in Korea [12].

Key Utility Decision Points: When alternative response strategies are available, the control room operators would probably request supervisory advice to choose the appropriate strategy. The representative example is the three possible post- SGTR cooldowns. When the required response actions are completed in an SGTR event after the identification of it, both radiological releases from the ruptured steam generator (SG) and primary-to-secondary leakage should be stopped. Additionally, the plant should be cooled and depressurized to a cold shutdown state. These cooldown and depressurization activities are complicated by the ruptured SG that will act as a second pressurizer and inhibit reactor coolant system (RCS) depressurization.

There are three alternative means for this post-SGTR cooldown: the backfill, blowdown, and steam dump method. The optimal method should be selected depending on the available plant equipment and the evolution of the event. In addition, the operator could execute combinations of the three methods at the same time. The decision-making procedure for automatically resolving this utility decision point is shown in Fig. 5. This decision-making procedure is developed on the basis of emergency response guideline background materials developed by the Westinghouse Owners Group [13] and the SROs' operational experience. 


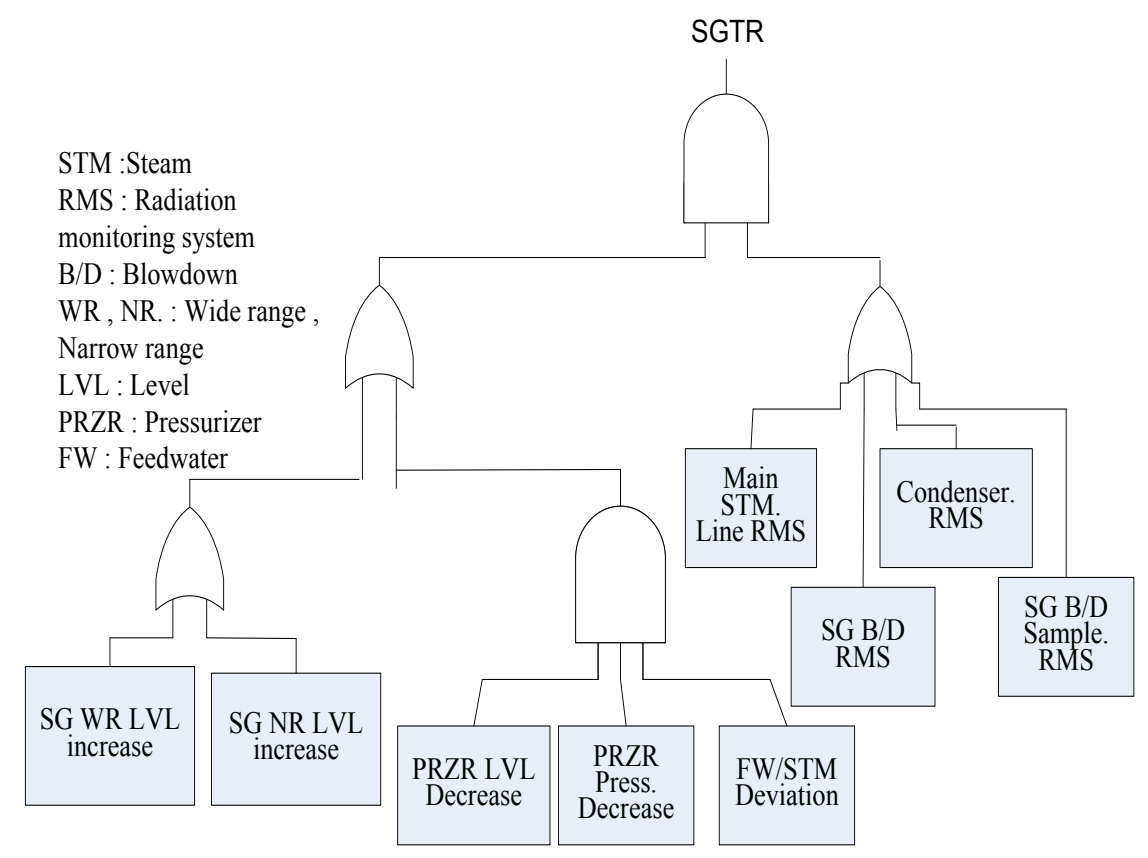

Figure 4. Diagnostic tree of an SGTR event.

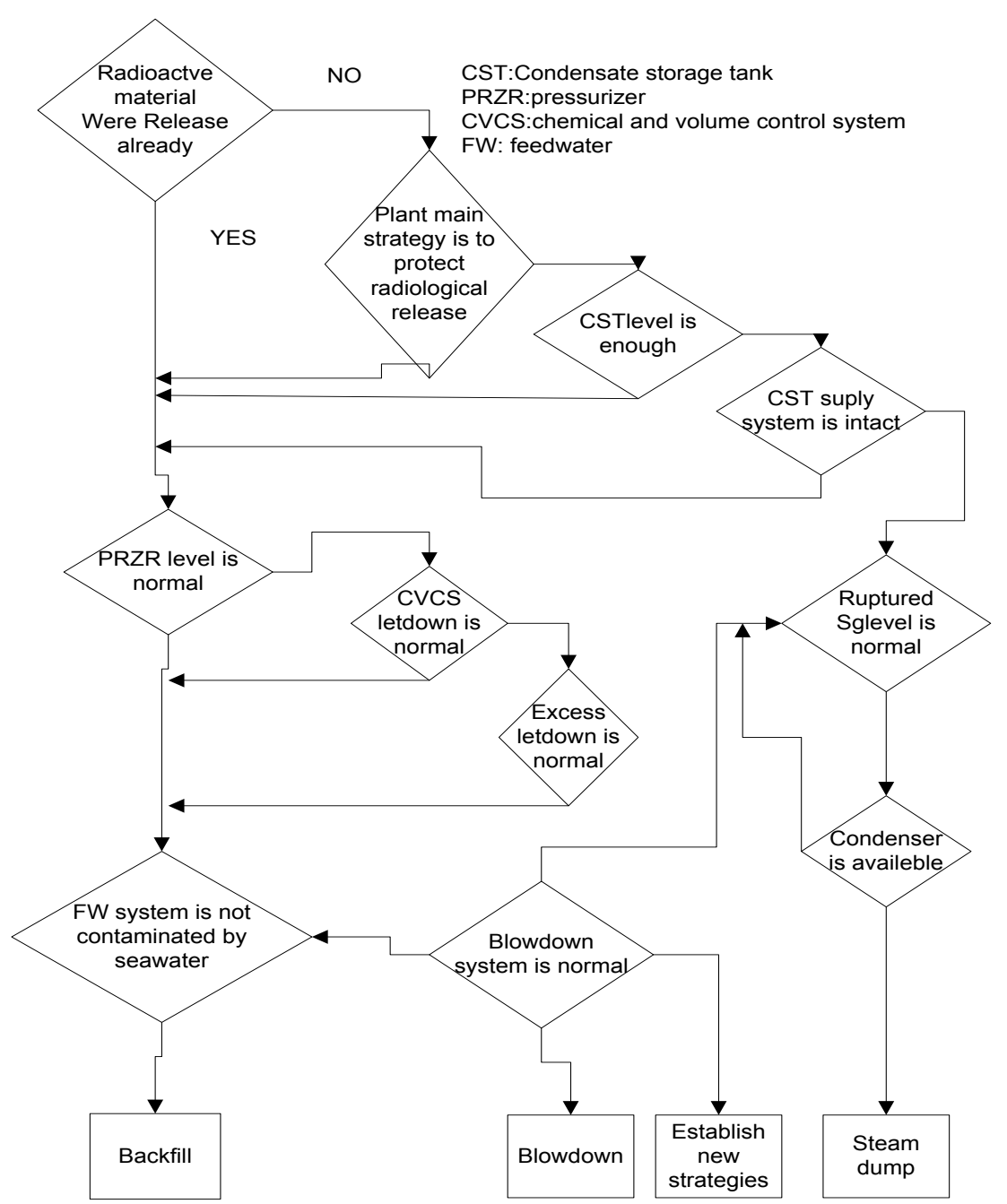

Figure 5. Decision-making procedure for selecting post-SGTR cooldown methods. 


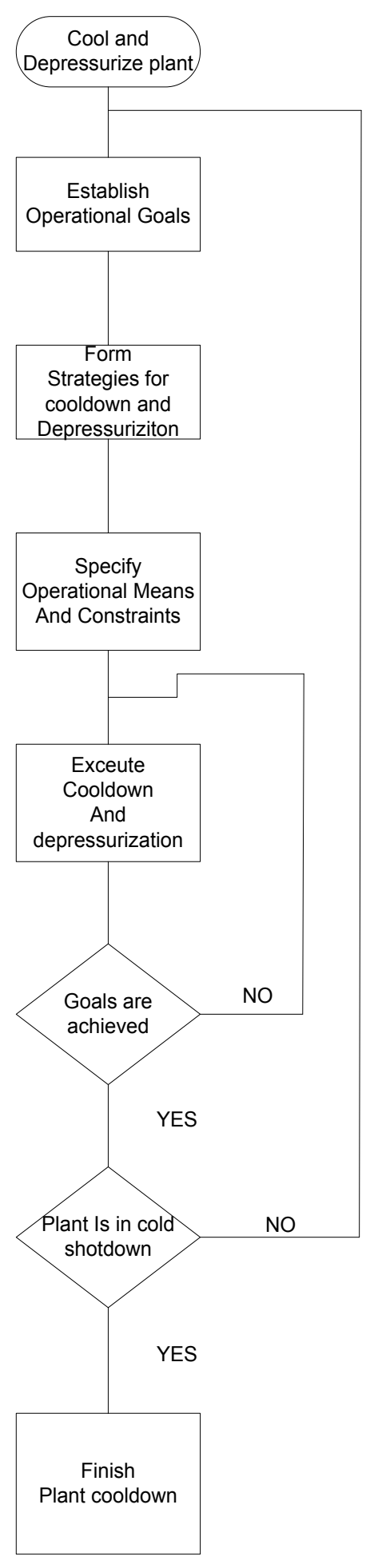

Figure 6. Operational sequence of the automated cooldown

\section{Control}

Analog controls are mainly necessary during the cooldown operation in emergency operation, the purpose of which is to cool and depressurize the plant to cold shutdown conditions in order to make repairs and ensure no further radiological releases. The controlled parameters for analog controls are as follows: RCS pressure, RCS temperature, pressurizer (PRZR) level, SG pressure, and SG level. Their operational constraints are related to subcooling operation, cooldown rate, PRZR level, and SG level. Among the controlled parameters, only the RCS pressure and temperature are treated in this section because their controls with tracking features are the most important to achieve optimal shutdown. The operational sequence of the automated cooldown is shown in Fig. 6. As shown in the figure, the operational goals are established by setting the goal values of the RCS pressure and temperature.

The fuzzy reasoning is used to reach the goals with the specification of operational means (BRU-A valve) and constraints(temperature margin\& cooldown rate). And then, cooldown and depressurization are iterated until the goals are achieved. This sequence is repeated until the plant reaches the cold shutdown conditions. The optimal shutdown strategies based on fuzzy reasoning are as follows.

- Structure of Fuzzy Reasoning: Fig. 7 shows the structure of fuzzy reasoning whose role is to decide the set points of the RCS pressure and temperature with a certain time interval $(30 \mathrm{~s})$ for the optimal shutdown. The optimal emergency operation is to locate the operational trace nearby the subcooling operation limit in the reactor coolant pressure versus hot-leg temperature curve ( $\mathrm{P}-\mathrm{T}$ curve), because this trace is desirable in view of maintaining reactor vessel integrity. As shown in Fig. 7, the generic structure consists of fuzzification, knowledge base, decision-making logic, and defuzzification.

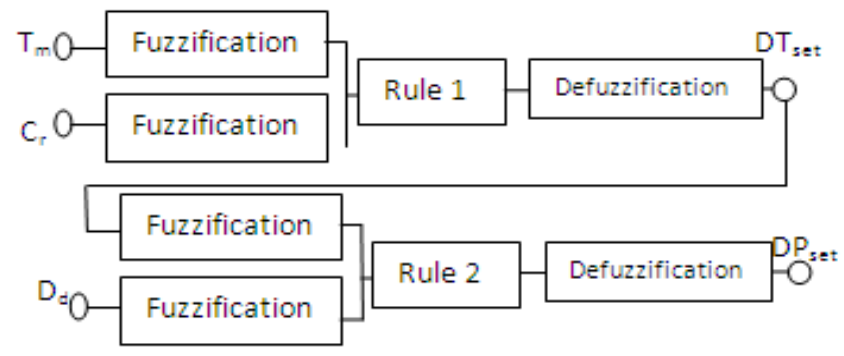

$\mathrm{T}_{\mathrm{m}}$ : Tempreture margin

$\mathrm{C}_{\mathrm{r}}$ : Cooldown rate

$\mathrm{D}_{\mathrm{d}}$ : Depressurization demand

$\mathrm{DT}_{\text {set }}:$ decrement of DT

$\mathrm{DP}_{\text {set }}$ : decrement of DP

Figure 7. Structure of generic fuzzy reasoning

- Fuzzification: Singleton fuzzification is used in this paper:

$$
\mu_{F}(X)=\left\{\begin{array}{l}
1 \rightarrow \text { if } X=X 0 \\
0 \rightarrow \text { if } X \neq X 0
\end{array}\right.
$$

The three input parameters for the fuzzy reasoning in order to decide the set points of the RCS pressure and temperature are as follows:

- temperature margin, $\mathrm{T}_{\mathrm{m}}: \mathrm{T}_{\text {sub }}-\mathrm{T}_{\text {hot }}$;

- cooldown rate, $\mathrm{C}_{\mathrm{r}}: \mathrm{dT} / \mathrm{dt}$;

- depressurization demand, $D d=\left[P-P_{\text {sub }}\left(T_{\text {set }}\right)\right] / D_{\mathrm{r}}$;

Where $\mathrm{T}_{\text {sub }}, \mathrm{T}_{\text {hot }}$ and $\mathrm{T}$ are the subcooling limit 
temperature at the $\mathrm{T}_{\text {hot }}$, the RCS hot-leg temperature, and the RCS average temperature, respectively, and $\mathrm{P}, \mathrm{P}_{\text {sub }}\left(\mathrm{T}_{\text {set }}\right)$ and $\mathrm{D}_{\mathrm{r}}$ are the RCS pressure, the subcooling limit pressure at the set point of the RCS temperature, and the depressurization rate, respectively.

The two output parameters for the fuzzy reasoning are defined as follows:

1) decrement of

$$
D T, D T \text { set }:\left\{\begin{array}{l}
D T(t)=D T(t-1)-D T \text { set } \\
T \operatorname{set}(t)=T(t)-D T(t)
\end{array}\right.
$$

2) decrement of

$$
D P, D P s e t:\left\{\begin{array}{l}
D P(t)=D P(t-1)-D P s e t \\
P s e t(t)=P(t)-D P(t)
\end{array}\right.
$$

where DT and DP are the decrements used to calculate the set points of the RCS temperature and pressure, and $\mathrm{T}_{\text {set }}$ and $\mathrm{P}_{\text {set }}$ are their calculated set points.

3) Knowledge Base: As shown in Fig. 8, first the set point of the RCS temperature is decided using the temperature margin and the cooldown rate, then the set point of the RCS pressure is decided considering the pressure drop due to RCS cooldown and the depressurization rate.

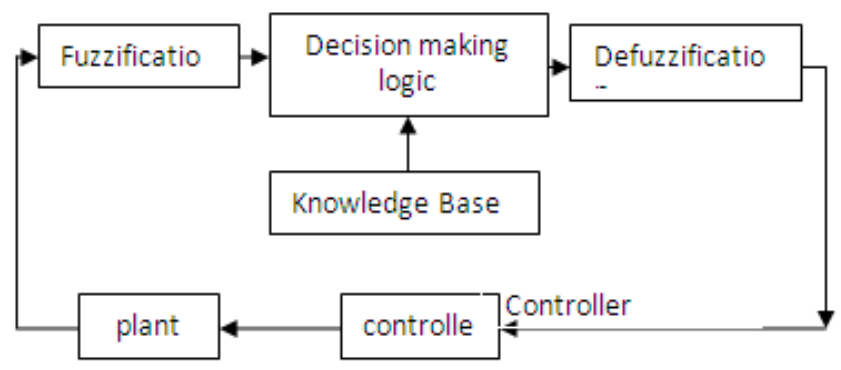

Figure 8. Fuzzy reasoning for optimal cooldown

The knowledge base transforms the three input parameters into linguistic variables that have their own linguistic values and triangular-type membership functions as shown in Fig. 9. The primary fuzzy set of the $\mathrm{T}_{\mathrm{m}}$ is categorized as small (S), medium (M), and large (L). The primary fuzzy set of the $C_{r}$ has six terms: very small (VS), small (S), slightly small (SS), medium (M), large (L), and very large (VL). That of the $D_{d}$ has seven terms: under large (UL), under medium (UM), under small (US), zero (Z), over small (OS), over medium $(\mathrm{OM})$, and over large (OL).

In the first step, all the input parameters are fuzzified using (1). Then, the $T_{m}$ and the $C_{r}$ are combined by Rule 1, which is tabulated in Table 1, to generate a new linguistic variable defined as the $\mathrm{DT}_{\text {set }}$. The terms of the $\mathrm{DT}_{\text {set }}$ are negative large $(\mathrm{NL})$, negative medium (NM), negative small (NS), zero (Z), positive small (PS), positive medium (PM), and positive large (PL). Then, the linguistic variable of the $\mathrm{DT}_{\text {set }}$ is defuzzified and fuzzified to generate a new linguistic variable defined as the $\mathrm{DP}_{\text {set }}$.
Table 1. Rule 1 For The Determination Of Dtset

\begin{tabular}{|c|c|c|c|}
\hline Tm Cr & S & M & L \\
\hline VS & PL & PL & Z \\
\hline S & PL & PM & NS \\
\hline SS & PM & PS & NM \\
\hline M & PM & Z & NM \\
\hline L & PS & NS & NL \\
\hline VL & Z & NM & NL \\
\hline
\end{tabular}

In the next step, the linguistic variable of the $\mathrm{DP}_{\text {set }}$ is generated by combining the $\mathrm{DT}_{\text {set }}$ and the $\mathrm{D}_{\mathrm{d}}$ by Rule 2 which is tabulated in Table 2. The $\mathrm{DP}_{\text {set }}$ has seven terms such as negative large (NL), negative medium (NM), negative small (NS), zero (Z), positive small (PS), positive medium $(\mathrm{PM})$, and positive large (PL), which have triangular membership functions as shown in Fig. 10.

Table 2. Rule 2 For The Determination Of Dpset

\begin{tabular}{|c|c|c|c|c|c|c|c|}
\hline $\begin{array}{c}D_{d} \\
\text { DTset }\end{array}$ & UL & UM & US & $Z$ & OS & OM & OL \\
\hline NL & NM & $Z$ & PS & PM & PL & PL & PL \\
\hline NM & NL & NS & $Z$ & PS & PM & PL & PL \\
\hline NS & NL & NM & $Z$ & $Z$ & PS & PM & PL \\
\hline Z & NL & NM & NS & $Z$ & PS & PM & PL \\
\hline PS & NL & NM & NS & $Z$ & $Z$ & PM & PL \\
\hline PM & NL & NL & NM & NS & $Z$ & PS & PL \\
\hline PL & NL & NL & NL & NM & NS & $Z$ & MP \\
\hline
\end{tabular}

4) Decision-Making Logic: The Inference Mechanism provides the mechanism for invoking or referring to the rule base such that the appropriate rules are fired. There are several methodologies. Two most common method used in fuzzy logic control are the maxmin composition and the max-product composition.we use the max-min method in this work.The rules in Tables I and II have the following format :

Rule $\mathrm{R}^{(\mathrm{i})}$ : IF $\mathrm{x}_{1}$ is $\mathrm{I}_{1}{ }^{(\mathrm{i})}$ and ... and $\mathrm{x}_{\mathrm{n}}$ is $\mathrm{I}_{\mathrm{n}}{ }^{(\mathrm{i})}$, THEN $\mathrm{y}$ is $\mathrm{O}^{(\mathrm{i})}$.

5) Defuzzification

The Centre Of gravity method is used for the defuzzification of the $\mathrm{DT}_{\text {set }}$ and the $\mathrm{DP}_{\text {set }}$.

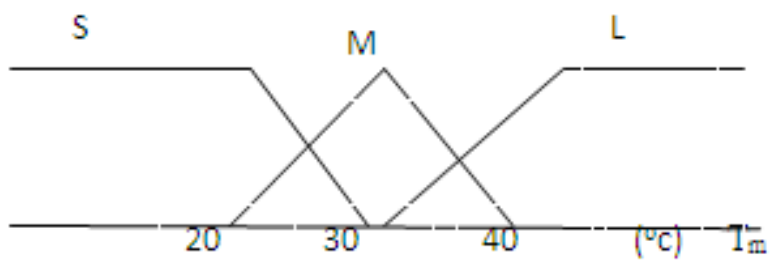

(a)

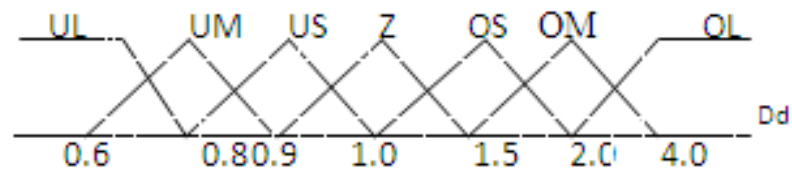

(b) 


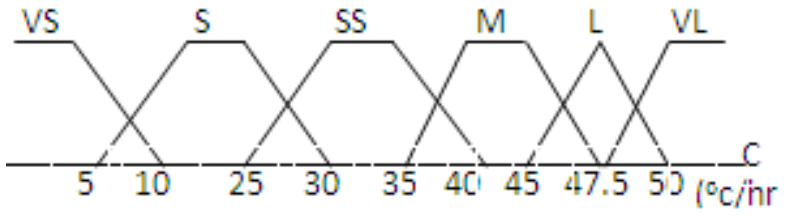

(c)

Figure 9. Membership functions for input parameters (a) Tm, (b) $\mathrm{Cr}$, and (c) Dd

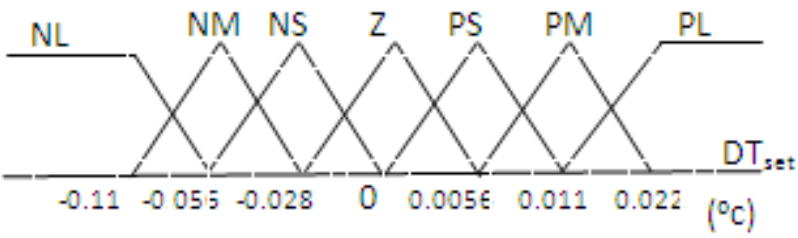

(a)

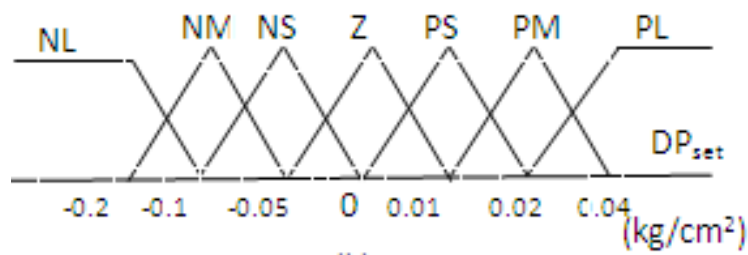

(b)

Figure 10. Membership functions for output parameters (a) DTset and (b) DPset

\section{Validation and Discussion}

Decision-Making

In order to verify the correctness of the established diagnostic trees, some tests were performed by using the microsimulator for the Kori Unit 2 [21], [22]. In these tests, nine events were simulated, and the activation of the diagnostic trees was observed as shown in Table III. Here, the numbers in the activated tree column correspond to the numbers indicating the simulated events. As indicated in the table, the diagnostic trees clearly discriminated corresponding events from the others in all the tests even though there were several partially activated trees in the case of some events.

Some experiments were performed using the real-time, fullscope simulator for Kori Units 3 and 4 to prove the validity of the decision-making procedure. In the experiments, eight main control room teams that consist of five or six operators were instructed to select the optimal post-SGTR cooldown method in the simulated scenario. Four teams among them were given the decision-making procedure while the others were not. The scenario was as follows: due to feedwater system contamination by sea water, the power reduction had been performed before the reactor trip from the SGTR event occurred. Then, the EOP for the SGTR was completed, and the SG B level was very high at that time [15]. In this scenario, the steam dump method is the most appropriate choice [14].

\section{Control}

The SGTR at the SG A has been chosen as an initiating event for the evaluation of the performance capability between manual controls based on the EOP's and automatic controls based on the fuzzy reasoning.

The SGTR scenario is caused by SG tube degradation which results from long operation or chemical/mechanical thermal stress and pressure shock [20]. If an SGTR occurs, the reactor coolant will be leaked to the secondary side because of the pressure difference between the primary and secondary side. Because the normal makeup system cannot compensate for the RCS leak, the emergency core cooling system supplies boric acid water. This prevents fuel rod failure by removing the residual heat and the decay heat from the fission product. While this accident is in progress, the ruptured SG should be isolated, and the primary pressure should be depressurized to the level of the ruptured SG pressure to minimize the break flow. Then safety injection should be terminated to prevent pressurizing the primary side again. After termination, the plant should be cooled and depressurized to cold shutdown conditions in order to make repairs and ensure no further radiological releases. In this evaluation, the steam dump method was applied to the post-SGTR cooldown.

The controlled and the controlling parameters, as well as the operational constraints, are all identified during an SGTR scenario through the EOP's analysis. The major parameters that should be controlled are as follows: RCS pressure, RCS temperature, PRZR level, and SG level. The operational constraints are: 1) emergency operation should be maintained in the subcooling range; 2) the cooldown rate should not exceed the limit of the technical specification to ensure reactor vessel integrity.

Table 3. Test Results of The Diagnostic Trees

\begin{tabular}{|c|c|c|c|}
\hline \multicolumn{2}{|c|}{ Simulated Event } & $\begin{array}{c}\text { Fully-activated } \\
\text { Tree }\end{array}$ & $\begin{array}{c}\text { Partially-activated } \\
\text { Tree } \\
\end{array}$ \\
\hline \multicolumn{2}{|c|}{ 1.Reactor trip response } & 1 & - \\
\hline \multicolumn{2}{|c|}{ 2.SI Termination } & 2 & $3,4,5,9$ \\
\hline \multirow{2}{*}{$\begin{array}{l}\text { 3. Faulted } \\
\mathrm{SG} \\
\text { isolation }\end{array}$} & $\begin{array}{l}\text { Main steam } \\
\text { line break }\end{array}$ & 3 & 4,5 \\
\hline & $\begin{array}{l}\text { Main FW } \\
\text { line break }\end{array}$ & 3 & $4,5,9$ \\
\hline \multirow{3}{*}{ 4.LOCA } & Small & 4 & 5 \\
\hline & Medium & 4 & 5 \\
\hline & Larg & 4 & 5 \\
\hline \multirow{3}{*}{ 5.SGTR } & $80000 \mathrm{~kg} / \mathrm{hr}$ & 5 & 4 \\
\hline & $100000 \mathrm{~kg} / \mathrm{hr}$ & 5 & 4 \\
\hline & $135000 \mathrm{~kg} / \mathrm{hr}$ & 5 & 4 \\
\hline \multicolumn{2}{|c|}{$\begin{array}{l}\text { 6.LOCA outside } \\
\text { containment }\end{array}$} & 6 & 4,5 \\
\hline \multicolumn{2}{|c|}{ 7.Loss of all AC power } & 7 & 4,5 \\
\hline \multicolumn{2}{|l|}{ 8.ATWS } & 8 & - \\
\hline \multicolumn{2}{|c|}{$\begin{array}{l}\text { 9.Loss of secondary heat } \\
\text { sink }\end{array}$} & 9 & $3,4,5$ \\
\hline
\end{tabular}


Table 4. Classification of Plant Operational Modes

\begin{tabular}{|c|c|c|c|c|}
\hline $\begin{array}{c}\text { Mode } \\
\text { Number }\end{array}$ & Description & Reactivity & $\begin{array}{c}\text { \%Rated } \\
\text { Thermal } \\
\text { power }\end{array}$ & $\begin{array}{c}\text { Average } \\
\text { Coolant } \\
\text { Tempreture }\end{array}$ \\
\hline 1 & $\begin{array}{c}\text { Power } \\
\text { operation }\end{array}$ & $\geq 0.99$ & $>10 \%$ & $\mathrm{~T}_{\text {avg }} \geq 176.7^{\circ} \mathrm{c}$ \\
\hline 2 & startup & $\geq 0.99$ & $\leq 10 \%$ & $\mathrm{~T}_{\text {avg }} \geq 176.7^{\circ} \mathrm{c}$ \\
\hline 3 & $\begin{array}{c}\text { Hot } \\
\text { Standby }\end{array}$ & $<0.99$ & 0 & $\mathrm{~T}_{\text {avg }} \geq 176.7^{\circ} \mathrm{c}$ \\
\hline 4 & $\begin{array}{c}\text { Hot } \\
\text { shutdown }\end{array}$ & $<0.99$ & 0 & $\begin{array}{c}100^{\circ} \mathrm{c} \leq \mathrm{T}_{\text {avg }}< \\
176.7^{\circ} \mathrm{c}\end{array}$ \\
\hline 5 & $\begin{array}{c}\text { Cold } \\
\text { shutdown }\end{array}$ & $<0.99$ & 0 & $\mathrm{~T}_{\text {avg }} \leq 176.7^{\circ} \mathrm{c}$ \\
\hline 6 & Refueling & $\leq 0.95$ & 0 & $\mathrm{~T}_{\text {avg }} \leq 176.7^{\circ} \mathrm{c}$ \\
\hline
\end{tabular}

The six-plant operational modes are recognized as shown in Table IV [18]. The emergency operation is executed in three stages. The first stage is from power operation to SI termination. The second stage is from SI termination to hot shutdown mode. The third stage is from hot shutdown to cold shutdown mode.

Manual Controls Based on the EOP's: In order to mitigate the consequences of an SGTR event, the controlled parameters are adaptively adjusted in the following way.

The RCS is cooled by steaming and feeding the intact SG, similar to a normal cooldown. Since all immediate safetyconcerns have been resolved, the cooldown rate should be maintained less than the maximum allowable rate of 60 $\mathrm{C} / \mathrm{h}$. This ensures more uniform fluid temperatures and minimizes the possibility of voiding in the primary system during depressurization.

BRU-A valves open on operable SG's and their transition into the cooldown mode at the rate of $60^{\circ} \mathrm{C} / \mathrm{h}$. Start-up of two pumps of additional boron injection for injection to PRZ to compensate for the secondary-to-primary leakage and to maintain the PRZR level on span .And isolation of affected SG 2 by the main, auxiliary and emergency feedwater and blowdown. MSIV closing on the steam line of affected SG.If the difference between saturation temperature at the core outlet and temperature in any hot leg of loops is reduced to $10^{\circ} \mathrm{C}$, Containment isolation valves are closed and and RCP set of all the loops are triped.

As the RCS temperature is reduced, the RCS is depressurized to promote backflow through the failed tube within the allowed subcooling operation range.

When the Coolant temperature at the reactor inlet is below $200^{\circ} \mathrm{C}$, the RCS temperature and pressure have been sufficiently reduced.Hence Terminate of RP emergency cooldown at the rate of $60^{\circ} \mathrm{C} / \mathrm{h}$. The residual heat removal (RHR) system cooling is initiated to complete the cooldown to cold shutdown. Once cold shutdown conditions are achieved, the PRZR can be cooled to terminate the event.

Fig. 11 illustrates the subcooling margin during manual controls based on the EOP's. Here, the break flow is $30 \%$ of 1 full tube rupture The break flows are chosen as the realistic values that can be established during SGTR scenarios .

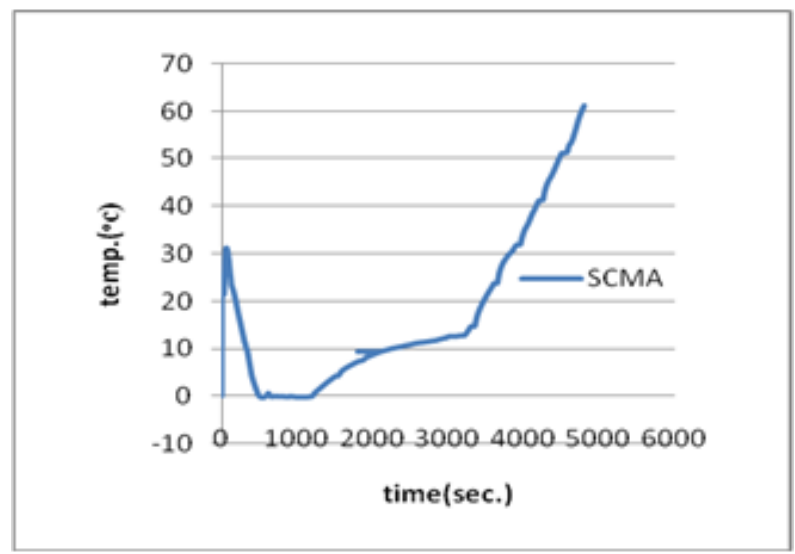

Figure 11. Subcooling margin during manual controls based on the EOP's

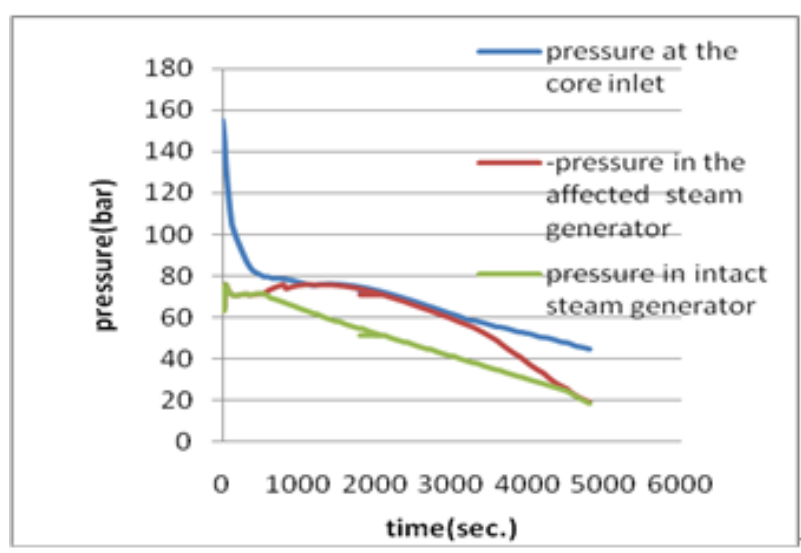

Figure 12. RCS pressure and the SG's pressures during manual controls based on the EOP's

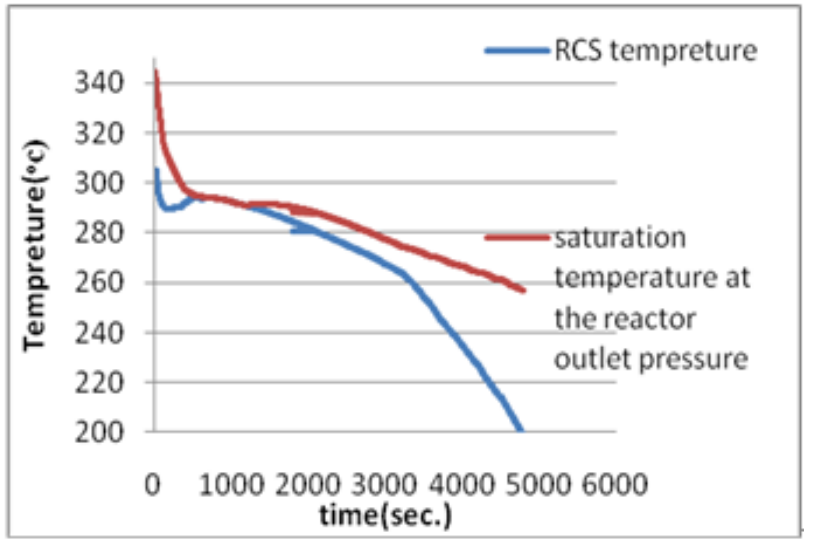

Figure 13. RCS average temperature manual controls based on the EOP's

Fig. 12 shows the trends of the RCS pressure and the SG's pressures during. The cooldown of the RCS average temperature manual controls based on the EOP's.is shown in Fig. 13, which shows that the cooldown rate was kept around the limit value of the technical specification $\left(60^{\circ} \mathrm{C} / \mathrm{h}\right)$ for the reactor vessel integrity.

Automatic Controls Based on the Fuzzy Reasoning: In order to optimize emergency operation during an SGTR 
event, the controlled parameters are adaptively adjusted in the following way. The RCS temperature and pressure are controlled by the proportional-integral (PI) unit. Their set points are set to the maximum allowable values within the subcooling operation range to minimize the break flow as soon as possible in the first operational stage.

They are decided through the fuzzy reasoning in the second stage as described in Section IV-B.

The proposed automating strategies were applied during SGTR scenarios by using the PCTRAN/VVER micro-simulator. A master module, which adjusts the set points of the controlled parameters, was implemented and integrated into the simulator in order to receive the plant operating variables and to feed the set points to the controllers.

Fig. 14 shows the $\mathrm{P}-\mathrm{T}$ curve to analyze temperature and pressure margin for the subcooling margin. As shown in the figure, all the emergency operations were performed within the allowed subcooling range in simulated case. the subcooling margin was maintained in the desired value $\left(30^{\circ} \mathrm{C}\right)$, which means these automated operation was much better than manual controls in Fig. 11 in view of reactor vessel integrity.

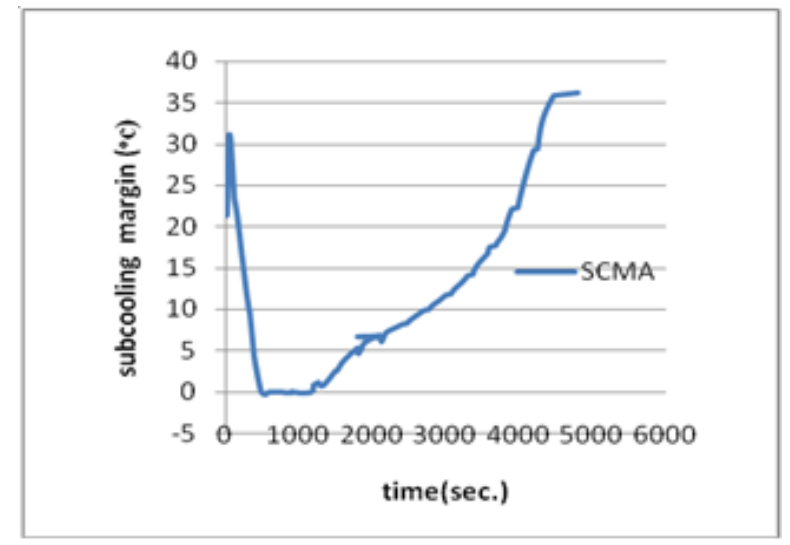

Figure 14. Subcooling margin during automatic controls based on the fuzzy reasoning

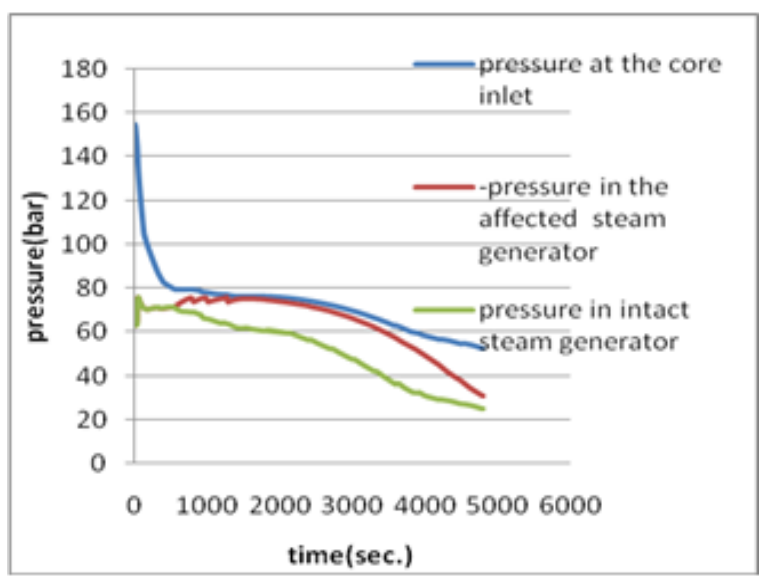

Figure 15. RCS pressure and the SG's pressures during automatic controls based on the fuzzy reasoning

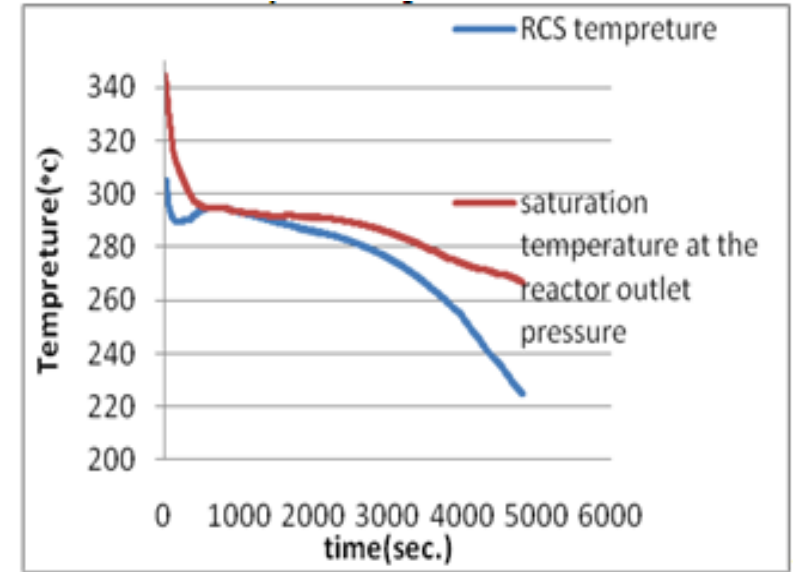

Figure 16. RCS average temperature during automatic controls based on the fuzzy reasoning

Fig. 15 shows the trends of the RCS pressure and the SG's pressures during automatic controls based on the fuzzy reasoning.

The RCS average temperature is shown in Fig. 16, which shows that the cooldown rate was kept below the limit value of the technical specification $\left(60^{\circ} \mathrm{C} / \mathrm{h}\right)$ for the reactor vessel integrity.

\section{Conclusions}

In this paper, the automating strategies of emergency operation are proposed for achieving optimal shutdown in a PWR(VVER 1000). These strategies are expected to considerably lengthen the operator response time as well as make emergency operation optimal in view of the material aspect of the reactor vessel.

The decision-making and control were investigated in order to develop the automating strategies.

From the control point of view, cooldown is planned by establishing operational goals in which the desired goal values of the RCS temperature and pressure are set, forming strategies by which the operational trace lies near the subcooling limit line within the allowed range in the $\mathrm{P}-\mathrm{T}$ curve and by specifying operational means and constraints. The reference set points of the controlled parameters are adaptively adjusted through the fuzzy reasoning according to the formed strategies for optimal cooldown and depressurization.

From the control point of view, cooldown is planned by establishing operational goals in which the desired goal values of the RCS temperature and pressure are set, forming strategies by which the operational trace lies near the subcooling limit line within the allowed range in the $\mathrm{P}-\mathrm{T}$ curve and by specifying operational means and constraints. The reference set points of the controlled parameters are adaptively adjusted through the fuzzy reasoning according to the formed strategies for optimal cooldown and depressurization. 
The validation of control automation was carried out with the SGTR scenarios. The results indicated that the automated emergency operation successfully drove the plant at full power to a cold shutdown state with all the operational constraints satisfied. In addition, the operational traces were much better than those of manual controls in view of reactor vessel integrity.

The results of this study are:

- Pressure in primary coolant system and steam lines of steam generators shall not exceed the design value by $15 \%$, that is, pressure of the coolant of the primary and secondary sides shall not exceed 20,24 and 9,02 MPa, respectively. As it seen from calculation results, the coolant pressure of the primary system does not exceed the initial value during the whole transient. Maximum coolant pressure of the secondary side is reached at the moment of 7,59 MPa In this case, the limiting values of coolant pressure of the primary and secondary sides are not reached, and the acceptance criterion for the given version is met. (Fig. 15)

- The analysis of calculation results has shown that values of maximum temperature of fuel and fuel rod cladding do not exceed their initial values during the whole accident process. Consequently, radially-averaged enthalpy of the fuel pellet does not also exceed its initial value (Fig. 17)

- No fuel rod damage.

- Maximum temperature of fuel pellet shall remain below the melting point of uranium dioxide (less than $2400^{\circ} \mathrm{C}$ for "burnt" fuel and respectively less than $2550{ }^{\circ} \mathrm{C}$ for "fresh" fuel); The accident considered is referred to the accidents with slow core reactivity variation, maximum fuel temperature does not exceed the initial value throughout the entire accident process.

- Radially-averaged enthalpy of fuel pellet do not exceed 840 and $963 \mathrm{~J} / \mathrm{g}$ for irradiated and fresh fuel, respectively.

- The primary coolant system is maintained in safe state, i.e. short- and long-term core cooling is ensured. design basis accident is analyzed from the beginning of the initiating event till the moment of reaching the parameters of design cooldown of the equipment under stable operation of safety systems. Calculation results show that throughout the entire transient the reactor core is flooded with cooling water, temperature of fuel and that of fuel rod cladding do not exceed the initial value.

- The initiating event can not result in more serious situation, (design basis accident will not result in more severe accident without additional failure). In other words, if additional failure is not imposed, the accident will not result in more severe accident in case the accident consequences are restricted by the limits established for such accidents.

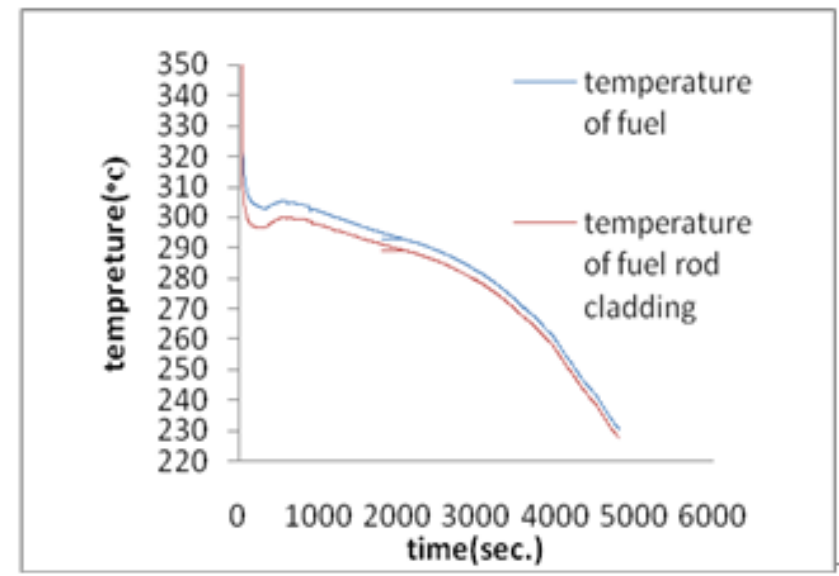

Figure 17. temperature of fuel and fuel rod cladding during automatic controls based on the fuzzy reasoning

\section{REFERENCES}

[1] Shimoda, H., Ishii, H., Wu, W., Li, D., Nakagawa, T., Yoshikawa, H., 1999. A basic study on Virtual Collaborator as an innovative human-machine interface in distributed virtual environment: the prototype system and its implication for industrial Application. IEEE SMC Conference Proceedings 5, 697-702.

[2] K. Iwaki, "Control room design and automation in the advanced BWR," in Proc./Ann. Int. Symp. Balancing Automation Human Action in Nuclear Power Plants, Munich, Germany, July 9-13, 1990, pp. 399-412.

[3] P. C. Goodman and C. A. Dipalo, "Human factors information system: Tool to assess error related to human performance in U.S. nuclear power plant," in Proc. Human Factors Soc. 35th Ann. Meeting, Oct. 1991, p. 662.

[4] J. A. Bernard and T. Washio, "Expert systems applications within the nuclear industry," in Proc. American Nuclear Soc., La Grange Park, IL, Oct. 1989.

[5] A. A. Husseiny, Z. A. Sabri, D. Packer, J. W. Holmes, S. K. Adams, and R. J. Rodriguez, "Operating procedure automation to enhance safety of nuclear power plants," Nucl. Engineering Design, vol. 110, pp. 277-297, 1989.

[6] W. C. Jouse and J. G. Williams, "Safety control of nuclear power operations using self-programming neural networks," Nucl. Sci. Eng., vol. 114, pp. 42-54, 1993.

[7] Y. Inazumi and M. Takashima, "Automatic control system for plant heatup and cooldown operations in Japanese PWR plants," in Int. Symp. NPP I\&C, Tokyo, Japan, 1992.

[8] K. Sekimizu and T. Araki, "Knowledge representation for automated boiling water reactor plant startup," Nucl. Technol., vol. 100, pp. 295-309, 1992.

[9] H. Yoshikawa, T. Magari, and Y. Yamamoto, "A review on progress of man-machine interface system designs for Japanese PWR's," Tech. Rep. Inst. Atomic Energy, Kyoto Univ. (Report no. 214), 1994.

[10] Korea Electric Power Corporation, "Emergency operating procedures of Kori Unit 2," Seoul, Korea, 1981. 
[11] S. S. Choi, K. S. Kang, H. G. Kim, and S. H. Chang, "Development of an on-line fuzzy expert system for integrated alarm processing in nuclear power plants," IEEE Trans. Nucl. Sci., vol. 42, pp. 1406-1418, Aug. 1995.

[12] S. H. Chang, K. S. Kang, S. S. Choi, H. G. Kim, H. K. Jeong, and C. U. Yi, "Development of the on-line Operator Aid SYStem OASYS using a rule-based expert system and fuzzy logic for nuclear power plants," Nucl. Technol., vol. 112, pp. 266-294, 1995.

[13] Westinghouse Owners Group, "Emergency response guidelines: Background volume for E-3 and ECA-3 guidelines," Westinghouse Electric Corp., PA, 1983.

[14] Final Safety Analysis Report for BNPP, chapter15 Accident Analysis, book 4, Revision 01 , Moscow(2007).

[15] Seong Soo Choi, Soon Heung Chang, , Durk Hun Lee, "Automating Strategies of Emergency Operation for Optimal Shutdown in Pressurized Water Reactors", IEEE Transactions on Nuclear Science 45,pp.17-29, 1998.

[16] Seung Jun Lee, Poong Hyun Seong, “ Development of automated operating procedure system using fuzzy colored petri nets for nuclear power plants" Annals of Nuclear Energy 31,pp. 849-869,2004.
[17] J. M. Izquierdo-Rocha and M. Sanchez-Perea, “Application of the integrated safety assessment methodology to the emergency procedures of a SGTR of a PWR," Reliability Eng. Syst. Safety, vol. 45, pp. 159-173, 1994.

[18] Korea Electric Power Corporation, "Final safety analysis report of Kori Unit 2," Seoul, 1981, pp. 16.1-16.7

[19] Abbasi ,s., Hadad,k. " Analysis of the loss of heat sink transients in the secondary circuit" of a VVER-1000 using RELAP5/MOD3.2 Annals of Nuclear Energy 47,pp. 28-37,2012.

[20] Gilbert Gedeon, P.E. "Thermal Shock and Brittle Fracture of Material, Department of Energy Fundamentals Handbook" Course No: T02-001 Credit: 2 PDH.

[21] J. J. Jeong and H. C. No, "An improved numerical scheme with the fully-implicit two-fluid model for a fast running system code," Nucl. Eng. Design, vol. 104, pp. 145-153, 1987.

[22] H. Seok, H. C. No, S. J. Cho, S. D. Park, H. Y. Jun, and Y. K. Lee, "Development of a software for the micro-simulator for Kori nuclear power plant unit 2,"Nucl. Technol., vol. 106, pp. 384-396, 1994. 